use of video consultations and improvement in communication before the virtual appointments. Our survey also shows that our patients have adapted well to virtual consultations and many are keen to have virtual consultation in the longer term. In our survey, only $6.8 \%(21)$ patients reported definite or clinically suspected COVID-19. Possible explanations for this include strict compliance with government advice on social distancing/shielding and limited testing at the onset of the pandemic. More patients assumed themselves to be clinically CEV than those who were actually CEV based on their treatment which is not surprising because of high level of anxiety among patients due to rapidly spreading pandemic and multiple sources of information.

This feedback provides useful data which will help us to plan the delivery of rheumatology services post COVID-19 pandemic. While face-to-face patient contact is needed for comprehensive disease assessment, teaching and training, a model for the future is likely to include a combination of face-to-face and virtual consultations. This could allow a greater capacity to see new patients and reduce waiting lists. Patients with uncomplicated and stable disease could be followed up in virtual clinics. There is also a need to formally incorportate the virtual consultations into the curriculum for Rheumatology trainees.

Disclosure of Interests: None declared.

DOI: 10.1136/annrheumdis-2021-eular.649

\section{POS1168 SELF-REPORTED SARS-CoV2 TESTING AND COVID-19 DISEASE IN PATIENTS WITH RHEUMATOID ARTHRITIS, AXIAL SPONDYLOARTHRITIS AND PSORIATIC ARTHRITIS IN A SWISS OBSERVATIONAL COHORT}

E. Papagiannoulis ${ }^{1}$, A. Ciurea ${ }^{2}$, D. Dan ${ }^{3}$, A. Finckh ${ }^{4,5}$, B. Gilbert ${ }^{4,5}$, I. Von Loga $^{1}$, C. Melong Pianta Taleng ${ }^{4,5}$, A. Scherer ${ }^{1}$, K. Lauper ${ }^{4,5,6} .{ }^{1}$ SCQM, Statistics, Zurich, Switzerland; ${ }^{2}$ Zurich University Hospital, University of Zurich, Rheumatology, Zurich, Switzerland ${ }^{3} \mathrm{CHUV}$, Rheumatology, Lausanne, Switzerland; ${ }^{4}$ Faculty of Medicine, University of Geneva, Internal Medicine, Geneva, Switzerland; ${ }^{5}$ Hôpitaux Universitaires de Genève (HUG), Rheumatology, Geneva, Switzerland; ${ }^{6}$ Centre for Musculoskeletal Research, University of Manchester, Centre for Epidemiology Versus Arthritis, Manchester, United Kingdom

Background: Since the beginning of the pandemic in Switzerland, immunosuppressed people were strongly advised to be tested for SARS-CoV2 when symptomatic as it was conjectured that they might be more at risk for infection and/ or severe disease. While patients with autoimmune diseases might be indeed more at risk of death from COVID-19 ${ }^{1}$, it remains unknown, whether there are differences in infection or complication rates between patients with rheumatoid arthritis (RA), psoriatic arthritis (PsA) and axial spondyloarthritis ( $\mathrm{AxSpA})$, and whether this relates to their disease or their treatment. Additionally, the prevalence of SARS-CoV2 testing in this population is not known.

Objectives: This study aimed to assess and compare the rate of COVID-19 and SARS-CoV2 testing in patients with RA, AxSpA and PsA, the potential association with their treatment and, for testing, the number of symptoms.

Methods: We included patients with RA, AxSpA and PsA from the Swiss Clinical Quality Management register (SCQM) using a smartphone app (mySCQM) to record information between March and December 2020. The outcomes of interest were self-reported SARS-CoV2 testing, symptoms compatible with COVID-19 during the previous month and confirmed COVID-19 through PCR nasopharyngeal swab. Outcomes were evaluated over the complete length of the aforementioned period (i.e. the outcome has been reported at least once during the period). Outcomes were compared between diseases groups, using logistic regression. We also evaluated the association of baseline treatment (TNF-inhibitors, b/tsDMARDs with other modes of action (OMA), no b/tsDMARDs) on the odds of symptoms and testing and the association of the number of symptoms (0-9) on the odds of testing. The analyses of SARS-CoV2 testing and COVID-19 symptoms were additionally adjusted for age, gender, glucocorticoids and csDMARDs. Confirmed cases were not adjusted for treatment and other covariates considering the low number of events.

Results: We included 927 patients with RA, 805 with AxSpa and 453 with PsA (Table 1). 1010 patients reported COVID-19-like symptoms (mostly fever, runny nose and cough), but only 455 of them (45\%) reported being tested. 151 patients were tested without symptoms. In between March and December 2020, 7.6\% of RA, $8.5 \%$ of AxSpA and $10.5 \%$ of PsA patients were tested positive for COVID-19 $(p=0.678)$. The odds of testing, symptoms and confirmed COVID-19 were similar between diseases and not associated with treatment for testing and symptoms (Figure 1). The number of symptoms was associated with the odds of testing (OR $1.43,95 \% \mathrm{Cl} 1.37-1.50$ by symptom)

Table 1.

\begin{tabular}{lccccc}
\hline & & RA & axSpA & PsA & p \\
\hline $\mathrm{n}$ & & 927 & 805 & 453 & \\
Age (mean, SD) & & $56.4(13)$ & $47.1(12)$ & $52.7(11)$ & $<0.001$ \\
Gender & female & $705(76)$ & $403(50)$ & $230(51)$ & $<0.001$ \\
Treatment & TNFi & $272(29)$ & $498(62)$ & $174(38)$ & $<0.001$ \\
& OMA & $355(38)$ & $71(9)$ & $137(30)$ & \\
& No b/tsDMARDs & $300(32)$ & $236(29)$ & $142(31)$ & \\
csDMARDs use & & $476(51)$ & $111(14)$ & $147(33)$ & $<0.001$ \\
Steroids use & & $93(10)$ & $11(1)$ & $19(4)$ & $<0.001$ \\
Disease duration & & $14.2(10)$ & $17.4(11)$ & $14.8(12)$ & $<0.001$ \\
Testing for COVID-19 & All patients & $258(28)$ & $231(29)$ & $117(26)$ & 0.550 \\
& Patients with & $189 / 427(44)$ & $179 / 392(46)$ & $87 / 191(46)$ & 0.911 \\
& symptoms & & & & \\
Presence of COVID-19 & & $427(46)$ & $392(49)$ & $191(42)$ & 0.082 \\
$\quad$ symptoms & & $18 / 237(8)$ & $19 / 223(9)$ & $11 / 105(11)$ & 0.678 \\
Positive COVID-19 test & & & &
\end{tabular}

$\mathrm{N}, \%$ when not specified otherwise. ${ }^{*}$ Missing data on test results in 41 patients $\chi 2$ test for categorical and t-test for continuous variables.

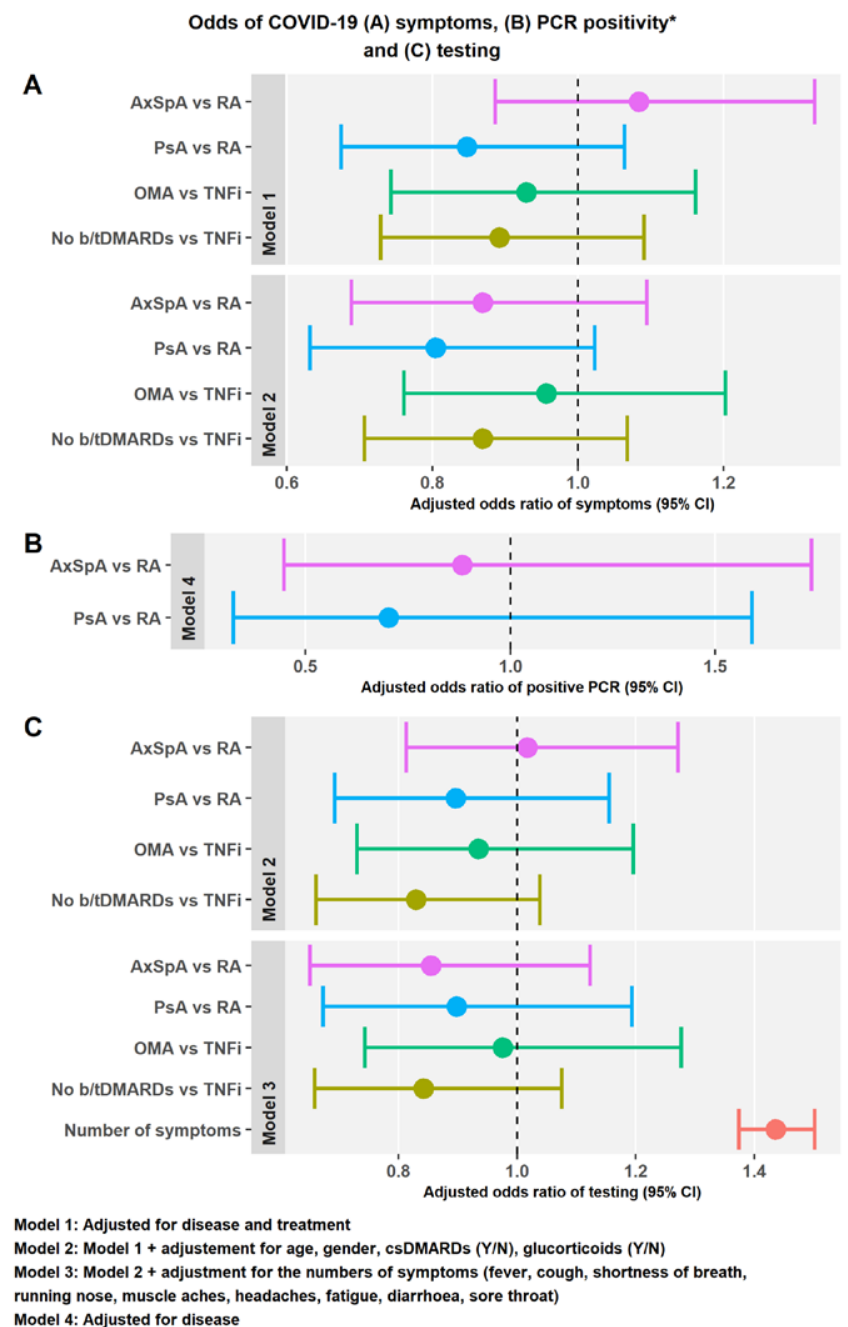

*In patients with information on tests results ( $\mathrm{N}=565$

Figure 1. 
Conclusion: Prevalence of COVID-19 symptoms and confirmed cases was similar between diseases, and for symptoms, was not associated with treatment. Despite strong advice from health authorities, less than $50 \%$ of patients with inflammatory rheumatic diseases and COVID-19 symptoms were tested. This proportion was not significantly different between diseases and not influenced by type of treatment. Efforts should be made to improve rates of SARS-CoV2 testing in patients with rheumatic diseases.

REFERENCES:

[1] Williamson, E. J. et al. Factors associated with COVID-19-related death using OpenSAFELY. Nature 584, 430-436 (2020).

Acknowledgements: This study was supported by Pfizer, Sanofi, Novartis, Gilead, Biogen and Bristol-Myers Squibb.

We thank all the patients and health professionals contributing to SCQM.

Disclosure of Interests: Eleftherios Papagiannoulis: None declared, Adrian Ciurea Speakers bureau: Abbvie, Eli Lilly, MSD, Novartis, Pfizer, Diana Dan: None declared, Axel Finckh: None declared, Benoit GILBERT: None declared, Isabell von Loga Consultant of: Deloitte Consulting AG., Cathy Melong Pianta Taleng: None declared, Almut Scherer Consultant of: Pfizer, Employee of: BMS (2007-2008), Kim Lauper Consultant of: Gilead Galapagos, Grant/research support from: AbbVie. The SCQM foundation is supported by different companies https://www.scqm.ch/en/sponsoren/.

DOI: 10.1136/annrheumdis-2021-eular.683

\section{POS1169 IMPACT OF THE COVID-19 PANDEMIC ON MORBIDITY AND MORTALITY AMONG SWEDISH PATIENTS WITH INFLAMMATORY JOINT DISEASES VERSUS THE GENERAL POPULATION}

H. Bower ${ }^{1}$, T. Frisell ${ }^{1}$, D. DI Giuseppe ${ }^{1}$, B. Delcoigne ${ }^{1}$, G. M. Alenius ${ }^{2}$ E. Baecklund $^{3}$, K. Chatzidionysiou', N. Feltelius ${ }^{4}$, H. Forsblad-D'elia ${ }^{5}$, A. Kastbom ${ }^{6}$, L. Klareskog ${ }^{1}$, E. Lindqvist ${ }^{7}$, U. Lindström ${ }^{5}$, C. Turesson ${ }^{8}$, C. Sjowall ${ }^{5}$, J. Askling ${ }^{1}{ }^{1}$ Karolinska Institutet, Department of Medicine Solna, Division of Clinical Epidemiology, Stockholm, Sweden: ${ }^{2}$ Umeå University, Rheumatology Unit, Department of Public Health and Clinical Medicine, Umeå, Sweden; ${ }^{3}$ Uppsala University, Department of Medical Sciences, Uppsala, Sweden; ${ }^{4}$ Swedish Medical Products Agency, Swedish Medical Products Agency, Uppsala, Sweden; ${ }^{5}$ Sahlgrenska Academy, University of Gothenburg, Department of Rheumatology and Inflammation Research, Gothenburg, Sweden; ${ }^{6}$ Linköping University, Department of Biomedical and Clinical Sciences, Linköping, Sweden; ${ }^{7}$ Skåne University Hospital, Lund University, Department of Clinical Sciences, Rheumatology, Lund, Sweden; ${ }^{8}$ Lund University, Rheumatology, Department of Clinical Sciences, Malmö, Sweden

Background: Studies from COVID-19 case-repositories among patients with rheumatic diseases have assessed associations (relative risks) between characteristics of the disease and adverse COVID-19 outcomes. Such designs are susceptible to bias from selection of cases reported. Few studies have assessed absolute and relative risks for COVID-19 outcomes in population-based cohorts of patients with inflammatory joint diseases, nor compared these risks to those in the general population.

Objectives: To estimate all-cause mortality, absolute and relative risks for severe COVID-19 in patients with chronic inflammatory joint diseases, compared over time and to the general population.

Methods: We updated a multi-register nationwide linkage ("ARTIS") on adults with RA, PsA, AS, SpA or JIA and population referents (matched on sex, age, and region), with data on hospitalizations, admission to intensive care (ICU), and deaths due to COVID-19. We calculated all-cause mortality March-September 2015-2020, and absolute and relative risks for COVID-19 outcomes March-September 2020. Patients were compared to population referents using hazard ratios (HR) from Cox models adjusted for comorbidities and socio-economy.

Results: We identified 110567 individuals with inflammatory joint disease (53455 with RA) in Sweden on March $1^{\text {st }} 2020$, and 484277 matched general population subjects. In all cohorts, the absolute risk of death from any cause in 2020 was higher than 2015-2019 (Figure 1), with a peak in mid-April, but the relative risks of death (vs. the general population) 2020 remained similar to HRs for 2015-2019 (HR for 2020 in Table 1).

Among all individuals with inflammatory joint disease in 2020, the risk for hospitalization, admission to ICU, and death due to COVID-19 was $0.5 \%$, $0.04 \%$ and $0.1 \%$, respectively (Table 1 ). HRs (vs. the general population) were elevated for almost all outcomes. HRs for COVID-19 related outcomes (Table 1) were higher than for non-COVID-19 outcomes; adjustment for co-morbidities and socio-economy explained much of these increases, somewhat less so for the former.

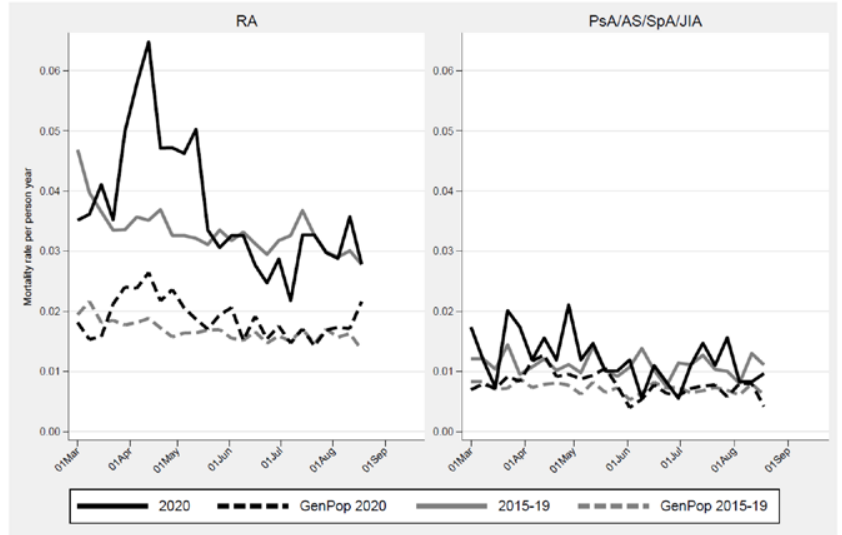

Figure 1. All-cause mortality in Swedish individuals with inflammatory joint disease and general population, March-September 2020 and the average 2015-2019

Table 1. Absolute and relative risks for COVID-19 outcomes in Swedish individuals with inflammatory joint disease compared to general population comparators March-September 2020

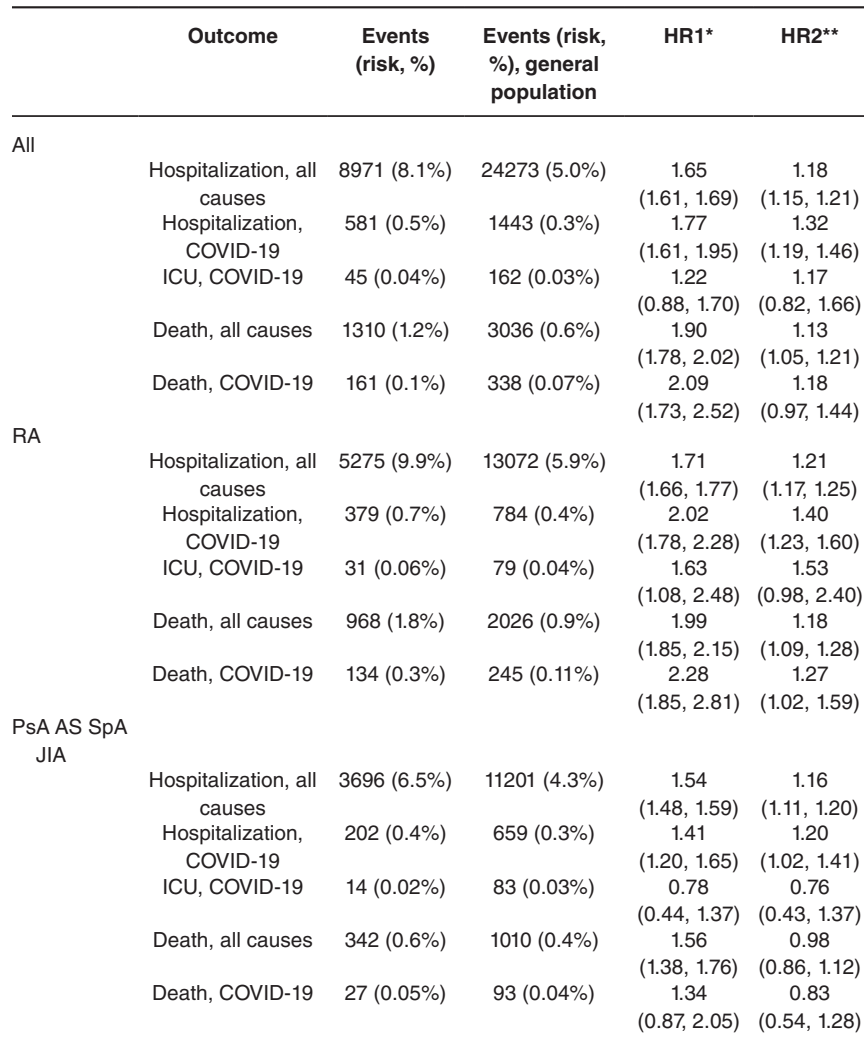

${ }^{*} \mathrm{HR} 1$ unadjusted, matched (age, sex, and region) ${ }^{* *} \mathrm{HR} 2$, as HR1 but adjusted for comorbidities and socio-economy

Conclusion: Risks of severe COVID-19 were increased among patients with inflammatory joint diseases, but similar increases were seen for non-COVID-19 morbidity. Co-morbidities and socio-economy explain much of this increase.

Disclosure of Interests: Hannah Bower: None declared, Thomas Frisell: None declared, Daniela Di Giuseppe: None declared, Bénédicte Delcoigne: None declared, Gerd-Marie Alenius: None declared, Eva Baecklund: None declared, Katerina Chatzidionysiou Speakers bureau: Eli Lilly, AbbVie and Pfizer, Consultant of: Eli Lilly, AbbVie and Pfizer, Nils Feltelius Employee of: Nils Feltelius is employed by the Medical Products Agency (MPA), which is a governmental body. The views in this abstract may not represent the views of the MPA, Helena Forsblad-d'Elia: None declared, Alf Kastbom Employee of: Former employee of Sanofi, Lars Klareskog: None declared, Elisabet Lindqvist: None declared, Ulf Lindström: None declared, Carl Turesson Speakers bureau: Roche, AbbVie and 